\title{
A Low Frequency Band for Video Watermarking base on PCA Technique
}

\author{
Deepti Pathak \\ Scholar M-Tech \\ Department of Computer Science and Engg. \\ Radharaman Institute of Technology and Science \\ Bhopal
}

\author{
Anurag Jain \\ Head \\ Department of Computer Science and Engg. \\ Radharaman Institute of Technology and Science \\ Bhopal
}

\begin{abstract}
As the internet users are increasing day by day because it is easy to transfer digital data between end users. By this new problem of data piracy has increased. There are different methods of watermarking has been proposed for protecting the digital data like video, audio, image, etc. There are number of researchers still working in the era of digital video watermarking from last few decades. This paper focuses on the video watermarking scheme for enhancing the privacy and authentication of a digital video during data transmission. Here the DWT and PCA techniques have been used. 2D DWT has been applied in LL part in order to enhance the robustness. With the help of modified cube selection approach, the water marks size and place of embedding is dynamic. The results show that proposed method gives the better results as compare to previous work.
\end{abstract}

\section{Keywords}

Digital Watermarking, DWT, frame segmentation, PCA

\section{INTRODUCTION}

With the growth in the digital electronics era most of the work get easy, one of them is transferring of data. But this technology rises a new problem of piracy or in other words proprietary get easily stolen. So to overcome this problem different techniques are use for preserving the proprietary of the owner, one of such digital approach is watermarking which is a subsection of hiding information that is used put some information in the original image which will specify the originality of the digital data like photographs, digital music, or digital video. One of the basic reason of the copyright issue is the ease available of the internet and some software that can modify the content as per the user requirement. Today bulk of information carries across the world on Internet. In recent year all the applications are moving towards the digital era, because of huge development in latest technologies such as in the area of communication, networked multimedia system, digital data storage etc. From the last two decades use of internet is rapidly enhanced in all environments towards achievement of effective, convenient and Security by introducing the digitization in their work. People regularly access web or network for e-mail, messaging, social networking, communication, business, transaction etc which help in growth and knowledge sharing in different domains such as education, research, development, Medical, and many business etc. Phenomenal development of Internet provides great facilities such as digital data can be copied without losing the originality and quantity of data. Speed and time saving are two main reason which help the users by using network or Internet and for this reason Internet is widely use. Digital watermarking is potential and robust solution of copyright issues. Digital watermarking is one of attention of researchers in copyright protection. A digital watermark also considered as digital signature which provides the one principle of security that is authentication. A given watermark may be unique for each copy (e.g. to identify the intended recipient) or it may be common for multiple copies. An owner can embed distinct watermarks in distinct objects to pinpoint the purchasers of the distinct copies of the protected material.



Fig1: Example of visible watermark in digital page.

Watermark is a kind of digital data in the form of text or image which can be store in the original signal. These text or image acts as the owner's signature in the data so that pirated and original data can be easily classified. As the pirated data do not have the original watermark this may be in form of text or image. Now watermarking technique is broadly classified into two fields first is visible watermarking while other is invisible watermarking.

Examples of visible watermarking in figure 1 and 2 are the digital page containing logo, T.V. channel contain logo of their channel etc.

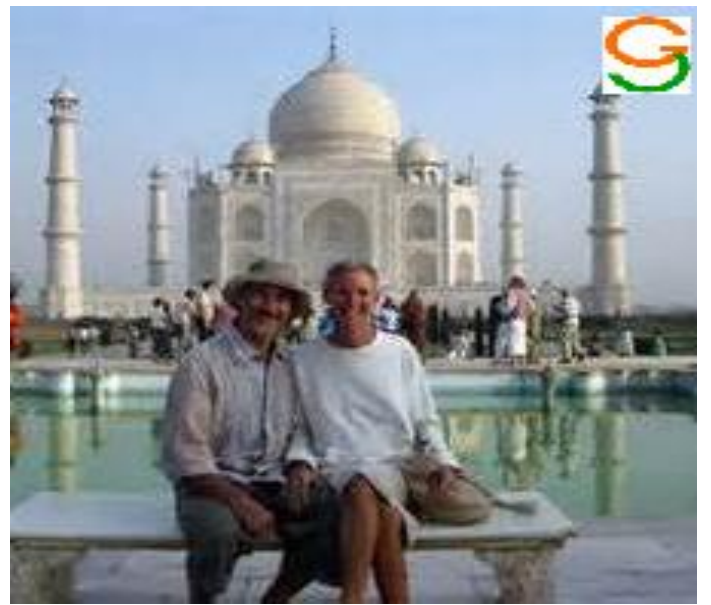

Fig2: Example of visible watermark in video. 
While for invisible watermark use by photographer, movies, etc. who put their watermark which is invisible while it contain the watermark data either in form of text or image. Most of the watermarking techniques focus on the invisible watermarking. As embedding the watermark into the digital data is quite tough and challenging although it is done by different methods.

\section{RELATED WORK}

In [1] Video Watermarking is done with empirical PCABased Decoding, here whole video is divide into different scenes then each frame is divided into blocks where combine blocks of that scene frames are considered as the cube. Now each cubes of the scene are analyze for cube selection in order to hide the watermark in the video. Once cube get select then with the help of 2-DWT and then PCA is applied for the insertion of watermark bits in the cube. This process is repeated with all the selected cubes. In case of De-Coding or extraction reverse process is applied as whole received video is divide into frames then scene are analyze then select cube and retrieve the watermark bit from it.

In [2] video watermarking process is done using side view. Here all frames are retrieved from the video then hold the side views of the frame and applied DWT on the side view. After this apply the Singular Value Decomposition (SVD) in the horizontal part of the DWT then embedded the watermark in this part and reconstruct the video frame in reverse order.

In [3] Digital Video Watermarking Technique is applied which is based on homogeneous Frame Extraction in 3-Level DWT. Here whole video is divide into sequence of frames then each similar sequence of frames are consider as the video shots, after this frames are convert into RGB format and then in the blue matrix of the frame 3-level DWT is apply. The matrix obtains after the third level of the horizontal part is utilized to store or embedded the watermark. After this inverse video watermarking is applied then put that frame in the same sequence and make the video in original form.

In [9] one more algorithm has developed using the PCA and DWT technique. Here divide the video frame and convert RGB frames in to YUV components. Then for each one frame select the luminance $\mathrm{Y}$ component and implement DWT to fragment the $Y$ frames into four different multiple resolution sub bands like LL, HL, LH, and $\mathrm{HH}$. After this divide the two sub bands LL and $\mathrm{HH}$ into $\mathrm{m} \times \mathrm{m}$ non overlapping partitions called as blocks and implement PCA to every block in the selected sub band LL and HH. Now embed the watermark into LL bands within the help of Discrete Wavelet Transform and Principal Component Analysis. For LL band, the watermark bits are embedded. At last apply inverse DWT is apply.

\subsection{Problem Statement}

With the increase in digital world, it is very easy for the transfer of the data from one place to other. But this lead new problem of preserving the proprietorship of the data. So watermark embedding is the solution for the digital video, one more issue is the invisibility of the watermark is also required. In previous work different techniques of watermarking is utilize where obtain results have very high Bit error rate and PSNR value is also very low. So a new combination of the DWT, PCA and Eludician distance is apply in the propose model for overcoming the problem of proprietorship maintain video quality by high PSNR value.
Previous work is not performed well in presence of different issues so overcoming of that problem is also required. Attacks like spatial and temporal attack on the video not generate fruitful watermark at the receiver side.

\section{PROPOSED WORK}

Whole work is divide into two steps first is embedding where video frames are analyze and watermark bits are embedded into the frames while in next step watermark bits are extract from the embedded video that is term as extraction. So figure 3 represents the various steps of embedding and figure 5 represents the extraction steps.

Original Video: As shown in block diagram first block will read original video. This reading means convert that video into collection of sequential frames. Now all frames are in form of two dimensional matrixes and complete video is of three dimensional matrixes. One dimension for row, second for the column and third for the frames.

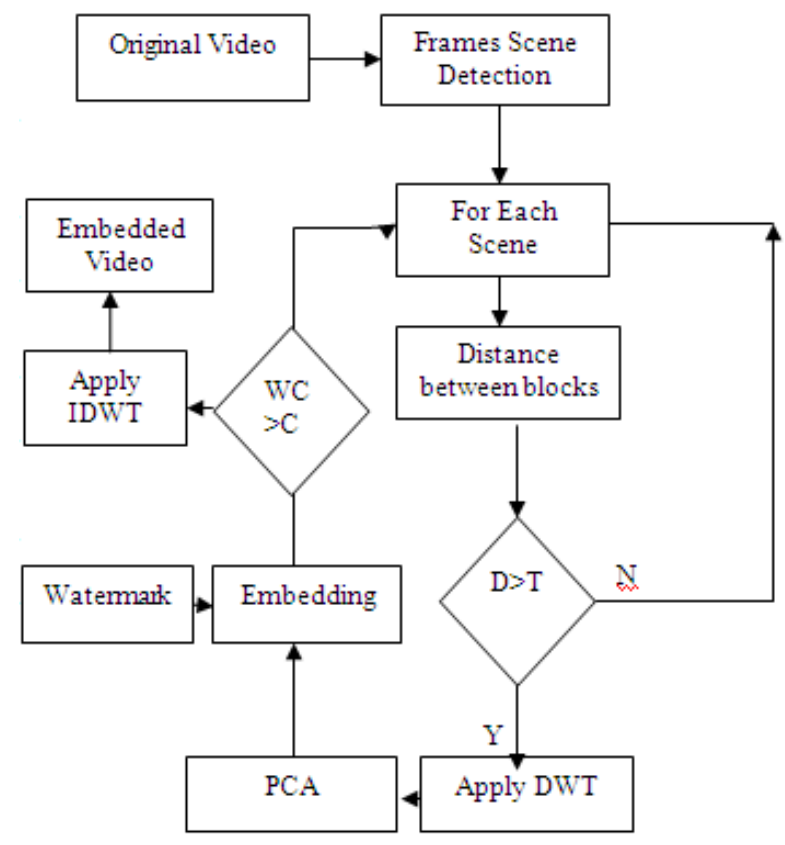

\section{Fig 3: Block Diagram of proposed Video Watermark Embedding Process}

\subsection{Frame Scene Detection}

In this block video is divided into scenes and the decision for selecting the scene is base on the edge detection algorithm. This will select those pixels from the frame which is present on the edges of the frame image. Now subtract those pixel values are required for the consecutive frames if these subtraction value is above the threshold value then new frame start from the current frame or put this frame in the same scene. Repeat this step for all the frames in the video.

\subsection{Scene Transformation}

Now each frame in the scenes is divided into equal size of blocks, where each scene looks like a collection of cube. Cubes are shown in figure 4 . Size of cube is range of pixel values such as of $8 \times 8,16 \times 16$, etc. Now each cube in the frame is examined for the evaluation purpose such that either that cube is eligible for embedding or not. This Selection of cube is depend on the difference of the pixel values of the block in the cube for the same if the difference is greater than some threshold value then it is selected for the embedding otherwise move towards the next cube. 


\subsection{Distance between Blocks}

This can be understand as Let $\mathrm{X}$ be a block of frame Fi from scene $\mathrm{S} 1$ and $\mathrm{Y}$ be the block of frame $\mathrm{Fj}$ from same scene then distance can be calculate as

$$
D=\sqrt{\operatorname{sum}\left((X-Y)^{2}\right)}
$$

\subsection{Discrete Wavelet Transform}

So if the cube is selected for the embedding then Apply DWT on it in actual portion of the matrix. As the frame is modified in the low frequency region so the effect of compression attack is very less. If direct change is done at this level then chance of watermark recovery get decrease and quality of video also degrade as at high frequency region human can detect it easily.

\subsection{Principal Component Analysis}

Next block of the work is to apply PCA on the selected cube after DWT. It generates new matrix for the cube of same size but as the work of PCA is to find the pattern that is obtain in this block.

This can be understand as if the selected matrix is $X$ then the resultant matrix is $\mathrm{Z}$ which is a multiple of the $\mathrm{X}$ and the transpose of the unit eigenvector of the sample covariance XXT , arranged in decreasing order of the corresponding eigenvalues, and $X$ is a centered version of $X$, with row means subtracted from each row.

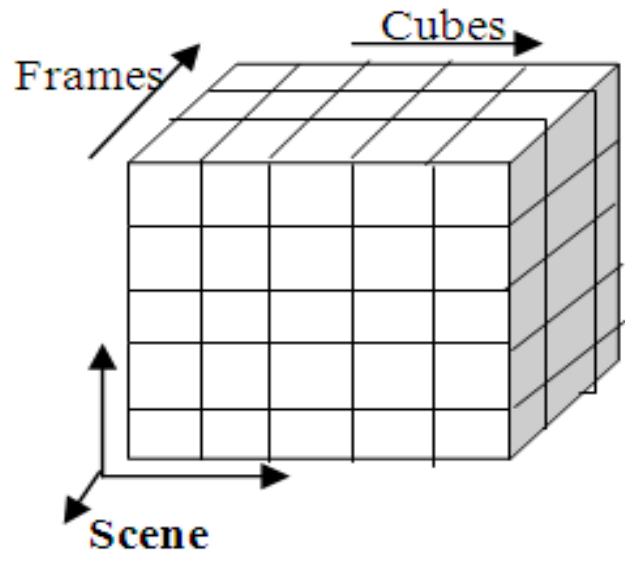

Fig4: Represents Cubes of the scene

\subsection{Embedding}

For embedding steps are simple, as matrix obtain from the PCA block are analyzed for the calculation of auxiliary bit. Here If matrix represents by $Z$ then read first row of $Z$ that is $\mathrm{z} 1:=(\mathrm{z} 1,1, \mathrm{z} 1,2 \ldots \mathrm{z} 1, \mathrm{~m})$. Now apply formula for the same matrix:

$$
\operatorname{Aux}=\left\{\begin{array}{l}
+1, y_{1, m / 2}-\frac{1}{m-1} \sum_{i=1, i \neq m / 2}^{m} y_{1, i}>0 \\
0, y_{1, m / 2}-\frac{1}{m-1} \sum_{i=1, i \neq m / 2}^{m} y_{1, i}<0
\end{array}\right\}
$$

Base on the above formula Aux value is either 1 or 0 . Now the aux bit and watermark bit are utilize for embedding, as the difference is need to create the embedding so a constant value is multiple and divide for the possible combination give below. Where constant represent by $\alpha$.

\subsection{Inverse Discrete Wavelet Transform}

Finally IDWT is apply on the embedded frame blocks this retransforms the matrix in original form. This is necessary as matrix is divided as per LL, HL, LH, HH quadrants. After IDWT all the blocks are combined back to single frame and then rejoin the frames into video

$$
X_{i, j}^{w}=\left\{\begin{array}{l}
\alpha \cdot x_{i, j},[j=m / 2, A u x=W=1 \\
\frac{1}{\alpha} \cdot x_{i, j},[j \neq m / 2, A u x=W=1 \\
\alpha \cdot x_{i, j},[j \neq m / 2, A u x=W=0 \\
\frac{1}{\alpha} \cdot x_{i, j},[j=m / 2, A u x=W=0
\end{array}\right\}
$$

Here as shown in block diagram all the steps of extraction are same as done in the embedding of the image. The only difference is in the extraction block. Here as watermark bit is not known so the calculation is reverse by estimating Aux value only.

$$
X_{i, j}^{w}=\left\{\begin{array}{l}
\frac{1}{\alpha} \cdot x_{i, j}, W=1,[j=m / 2, A u x=1 \\
\alpha \cdot x_{i, j}, W=1,[j \neq m / 2, A u x=1 \\
\frac{1}{\alpha} \cdot x_{i, j}, W=0,[j \neq m / 2, A u x=0 \\
\alpha \cdot x_{i, j}, W=0,[j=m / 2, A u x=0
\end{array}\right\}
$$

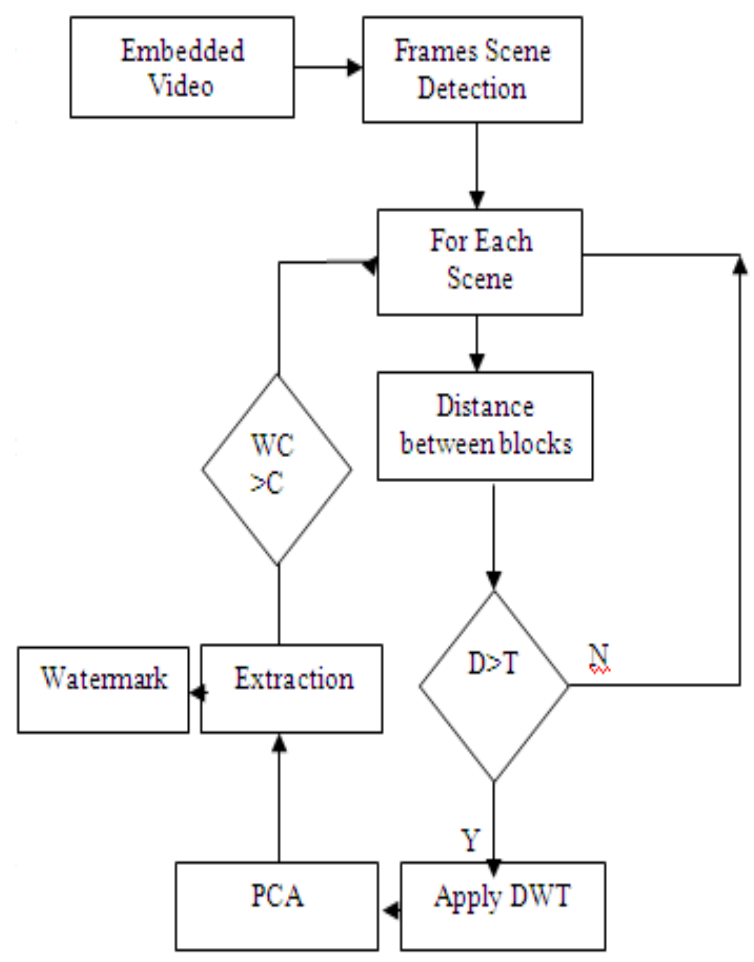

Fig5: Represent the block diagram of Extraction Extraction Steps

\section{EXPERIMENT AND RESULT}

This section represents the experimental analysis and evaluation of the proposed embedding and extraction technique for achieving privacy of a video. Experiment is done on the video that are of different environment. 


\subsection{Evaluation Parameter}

\subsubsection{Peak Signal to Noise Ratio}

It is the ratio between the maximum possible received information and the noise that affects the fidelity of its representation

$$
\mathrm{PSNR}=10 \log _{10}\left(\frac{\text { Max_pixel_value }}{\text { Mean_Square_error }}\right)
$$

\subsubsection{Bit Error Rate}

In this parameter one can obtain the ratio of number of subtraction of total watermark bit by correct watermark bits are read after the extraction to the total watermark bit.

BER is zero means no error is obtain or all the watermark bits are successfully retrieve.

$$
\frac{\text { Total_Watermark_Bit-Correct_Watermark_bit }}{\text { Total_Watermark_Bit }}
$$

Table 1. Represents base Paper [1] and Proposed work comparison on different Environment.

\begin{tabular}{|c|c|c|}
\hline \multicolumn{3}{|c|}{ Water Mark Positions } \\
\hline Environment & Proposed work & Base Paper [1] \\
\hline Jungle & 48928 & 5981 \\
\hline College & 70800 & 9396 \\
\hline
\end{tabular}

As table 1 shows that number of space in the same video for watermarking is more in proposed work while in case of base paper [1] it is quite low. Same results obtain in different video and in different environment which shows that proposed work can hold large amount of data.

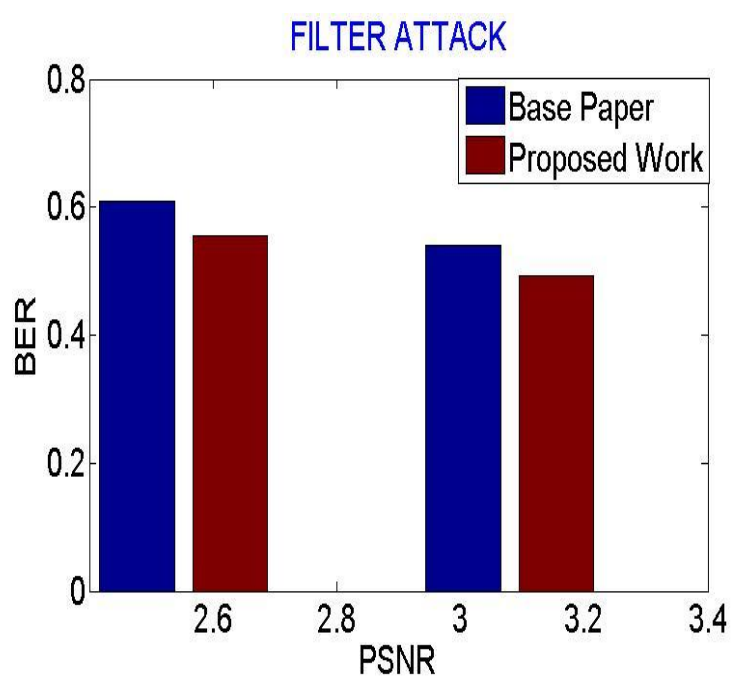

Fig6: Represents base paper [1] and Proposed work comparison on Jungle video.

Figure 6 represents the filter attack on the previous work in [1] which is term as base paper and proposed work, it is obtain that proposed work give excellent result in Gaussian noise attack.



Fig7: Represents base paper [1] and Proposed work comparison on College video.

Figure 7 represent the noise attack on the previous work in [1] which is term as base paper and proposed work; it is again obtain that proposed work give excellent result in Gaussian filter attack. As the video environment is different still proposed method works well.

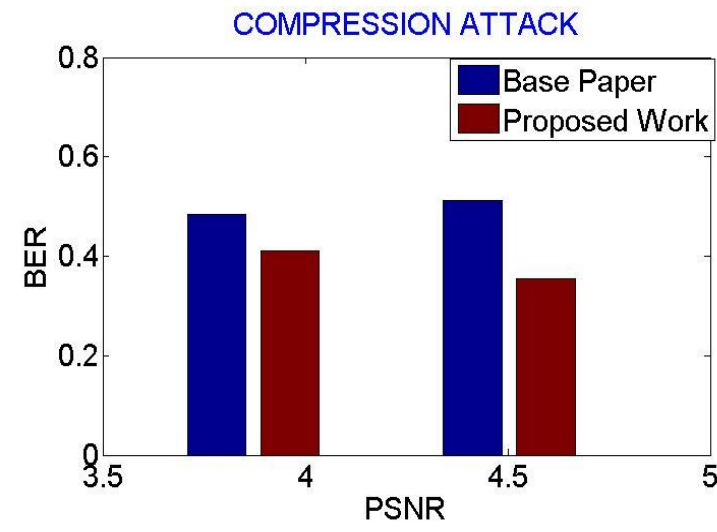

Fig8: Represents base paper [1] and proposed work comparison on College video.

Figure 8 represent the Compression attack on the previous work in [1] which is term as base paper and proposed work, it is again obtain that proposed work give excellent result in Gaussian filter attack. As the video environment is different still proposed method works well.

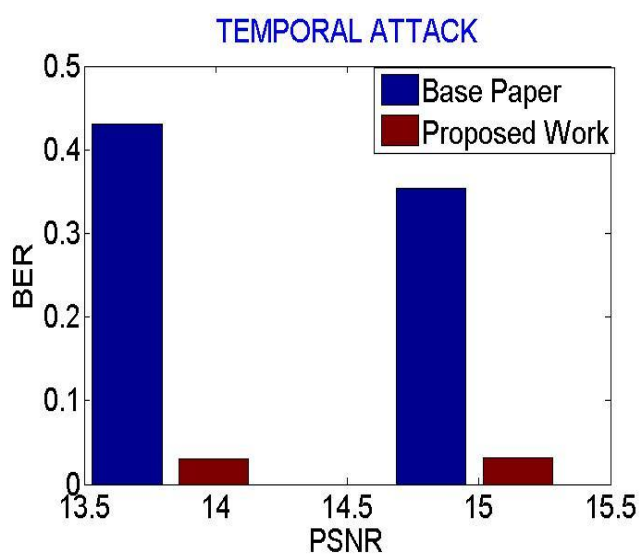

Fig9: Represents base paper [1] and proposed work comparison on Jungle video 
Figure 9 represents the temporal attack on the previous work in [1] which is term as base paper and proposed work; it is again obtain that proposed work give excellent result in Gaussian filter attack. As the video environment is different still proposed method works well.

\section{CONCLUSION}

In this paper a new approach of watermarking is implemented where frequency domain is utilized for the work. Proposed work is evaluated on the different attacks at different levels such as spatial and geometrical. Results show that proposed work is well in all conditions with various parameters. It has been obtained that 0.03 is the BER rate of the work in presence of attack condition which is high effective. There is need to work in research so the system need improvement in cropping attack and rotation attack as well.

\section{REFERENCES}

[1] Hanieh Khalilian, Student Member, IEEE, and Ivan V. BajicVideo "Watermarking With Empirical PCA-Based Decoding" IEEE TRANSACTIONS ON IMAGE PROCESSING, VOL. 22, NO. 12, DECEMBER 2013.

[2] Walter Godoy Jr., Charles Way Hun Fung “ A novel DWT-SVD video watermarking scheme using side view" 978-1-4577-1180-0/11/\$26.00 @2011 IEEE.

[3] Tamanna Tabassum, S.M. Mohidul Islam "A Digital Video Watermarking Technique Based on Identical Frame Extraction in 3-Level DWT" vol. 13, no. 7, pp. $560-576$, july 2003.
[4] Frank Hartung, Jonathan K. Su, and Bernd Girod "Spread Spectrum Watermarking: Malicious Attacks and Counterattacks". of Multimedia Contents" International Journal of Research in Engineering and Technology eISSN: 2319-1163 | pISSN: 2321-7308

[5] "CHAPTER 2. WAVELET TRANSFORMS ON IMAGES" sundoc.bibliothek.uni-halle.de/dissonline/02/03H033/t4.pdf

[6] A.F. ElGamal, N.A.Mosa , W.K.ElSaid A Fragile Video Watermarking Algorithm for Content Authentication based on Block Mean and Modulation Factor International Journal of Computer Applications (0975 - 8887) Volume 80 - No.4, October 2013.

[7] Nallagarla.Ramamurthy\#1 and Dr.S.Varadarajan. "Effect of Various Attacks on Watermarked Images. "International Journal of Computer Science and Information Technologies, Vol. 3 (2) , 2012,3582-3587

[8] Priya Porwal1, Tanvi Ghag2, Nikita Poddar3, Ankita Tawde DIGITAL VIDEO WATERMARKING USING MODIFIED LSB AND DCT TECHNIQUE. International Journal of Research in Engineering and Technology eISSN: 2319-1163.

[9] Mr Mohan A Chimanna 1,Prof.S.R.Kho "Digital Video Watermarking Techniques for Secure Multimedia Creation and Delivery" Vol. 3, Issue 2, March -April 2013, pp.839-844839. 